\title{
3 Methodisches Vorgehen
}

Zusammenfassung: Im Kapitel werden die methodischen Prämissen, die sich für die Identifizierung eines sprachhistorischen Inventars kolonial motivierter Straßenbenennungen zwischen 1884 und 1945 ergeben, dargelegt. Zur systematischen, ortsübergreifenden Erhebung der bislang unerforschten kolonialen Straßennamenvergabepraktiken ist eine empirisch verankerte, streng korpusbezogen nach etablierten sprachwissenschaftlichen Methoden analysierende Methodik notwendig. Das umfangreiche, materialbasierte Identifizierungsverfahren der additiven Informationsgewinnung aus unterschiedlichen Quellen und Quellengattungen wird exemplarisch anhand von zwei Städtebeispielen des erstellten Ortskorpus erläutert. Daran können auch die unterschiedlichen Blickrichtungen (ex ante vs. ex post) auf den thematischen Zusammenhang von Kolonialismus und Straßennamen erneut herausgestellt werden: Sie sind die Ursache dafür, dass sich das in dieser Studie analysierte sprachhistorische Nameninventar zum Teil von den rezent in Umbenennungsdiskussionen diskutierten und als ,kolonial bezeichneten Straßennamen unterscheidet.

\subsection{Grundprinzipien}

Die Untersuchung konstitutiver Sprachgebrauchsmuster kolonialer Raumaneignung bzw. -besetzung in der deutschen Metropole resultiert aus einem sprachhistorischen Interesse, also aus einer Perspektive ex ante (vgl. Warnke et al. 2019: 1). Die Arbeit setzt sich zum Ziel, im empirisch-systematischen Zugriff ein ortsübergreifendes Namenkorpus kolonial motivierter Straßennamen zusammenzustellen, die bis 1945 in den (Alltags-)Raum des Deutschen Reichs verfügt wurden. Die Erstellung des Nameninventars erfolgt damit in einem historischen und zugleich ortsübergreifenden Zugriff mittels deskriptiv-analytischer Vorgehensweisen. Jene drei formulierten Grundprinzipien werden in Kap. 3.1 näher erläutert. In Kap. 3.2 wird das erstellte Ortskorpus beschrieben, welches hinsichtlich etwaiger kolonial motivierter Straßenbenennungen untersucht wurde. In Kap. 3.3 werden die hierfür notwendigen Quellengattungen vorgestellt und diskutiert. Anhand von zwei ausgewählten Ortspunkten werden die Arbeitsschritte jener Inventarisierungsaufgabe in Kap. 3.4 aufgezeigt. Dabei wird deutlich, dass sich die vorliegende Studie sowohl von Arbeiten in den Postcolonial Language Studies mit einer vorab gesetzten kritischen Theoriebildung als auch von gegenwärtigen Ansätzen gesellschaftspolitischer Akteure (sog. ,postkolonial.de‘-Gruppen) unterscheidet, die rezente Straßennamen in kritischer Intention zur Diskussion stellen.

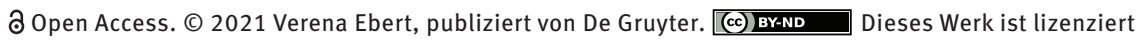
unter der Creative Commons Attribution-NoDerivatives 4.0 International Lizenz. https://doi.org/10.1515/9783110718133-004 


\subsubsection{Sprachhistorischer Zugriff}

Bei der Untersuchung von Kolonialtoponymen sind „Kontextgebundenheit und historische Faktizität von kolonialzeitlichen Daten [...] unhintergehbar“ (Stolz und Warnke 2018b: 51). Die Beantwortung der in dieser Arbeit zentralen Leitfrage, welche kolonisatorischen Wissensbestände zwischen 1884 und 1945 mittels kolonial motivierter Straßenvergabepraktiken in den (Alltags-)Raum des Deutschen Reichs versprachlicht wurden, setzt voraus, dass die zwischen 1884 und 1945 erfolgten Benennungen quellenbezogen, also aus einer sprachgebrauchsgeschichtlichen Perspektive ex ante identifiziert und sodann inventarisiert werden. Nur auf einer solchen Datengrundlage können linguistische Kommentierungen und daran anschließende Analysen ,nach historischen Selbstverständlichkeiten, nach historisch geteiltem Wissen, nach dem, was einer Gesellschaft bzw. Teilgruppe als common sense galt“ (Warnke et al. 2016: 20), erfolgen. Ein Straßenname ist folglich nur dann als ,kolonial motiviert` $\mathrm{zu}$ beurteilen und in das zu erstellende Namenkorpus aufzunehmen, wenn der jeweilige Name zum Zeitpunkt seiner historischen Verfügung bis 1945 in einem unmittelbaren Zusammenhang mit der deutschen Kolonialgeschichte verortet werden kann. Die Erhebung eines solchen Nameninventars kann nicht durch primär strukturelle Kriterien im Sinne eines festgelegten Sets von Zeichenfolgen geleistet werden, denen kolonial motivierte Benennungen folgen. Vielmehr handelt es sich um eine inhaltsseitige Zuschreibung des Namens zum historischen Vergabezeitpunkt in Bezug auf Kolonien und koloniale Themen, die bei der Katalogisierung jedes einzelnen SN-Tokens als einzelne sprachliche Äußerung überprüft werden muss. Damit ist ein methodisches Vorgehen nötig, mit dem im systematischempirischem Zugriff SN-Token zusammengestellt werden können, die zum Zeitpunkt ihrer historischen Verfügung zwischen 1884 und 1945 von den städtischen Administrationen tatsächlich im unmittelbaren kolonialen Kontext sprachlich verortet wurden.

\subsubsection{Ortsübergreifender Zugriff}

Die bisherigen singulären Studien aus den Geschichts- und Kulturwissenschaften setzten sich einzelortsbezogen unter anders gelagerten Fragestellungen mit kolonialen Straßennamen auseinander. Sie liefern nur erste Anhaltspunkte dafür, dass die Etablierung kolonialen Ehrens im öffentlichen (Alltags-)Raum durch entsprechende Straßennamenverfügungen für die faktische Kolonialepoche in der Zeit des Kaiserreichs, darüber hinaus aber für einzelne Städte auch in den 1920er und 1930er Jahren festzustellen ist. Bis dato ergibt sich ein völlig 
ausschnitthaftes Bild, mit denen die Frage nach den mit derartigen Benennungspraktiken versprachlichten ortsübergreifend-nationalen Gewissheiten nicht beantwortet werden kann. Diese setzt eine nach historischen Kriterien geleitete systematische Erhebung eines ortsübergreifenden Nameninventars voraus. Erst dann sind Analysen hinsichtlich etwaiger nationaler sprachlicher Interessen möglich. Der zugleich in onomastischen als auch in geschichts- und (weiteren) kulturwissenschaftlichen Studien eingeforderte ortsübergreifendvergleichende Zugriff wird in der vorliegenden Studie erstmalig umgesetzt. Um ein kolonial motiviertes Inventar auf einer vergleichsweise hohen Stichprobe zusammentragen zu können, ist die systematische Erstellung eines Ortskorpus notwendig, das kriterienbasiert aus der Perspektive ex ante zu erfolgen hat und eine möglichst große Zahl an Städten umfasst, die zwischen 1884 bis 1945 zum Deutschen Reich gehörten. ${ }^{55}$

\subsubsection{Deskriptiver Zugriff}

Den zuvor beschriebenen sprachhistorischen und ortsübergreifenden Zugriffen ist ein maßgebliches drittes Grundprinzip hinzuzufügen: Der innerhalb der modernen Sprachwissenschaft präferierte deskriptive Anspruch (vgl. Klein 2004: 376-377) wurde von Warnke et al. (2016: 10-14) für die Postcolonial Language Studies erläutert: Koloniallinguistische Studien arbeiten zeitgleich zu aktuellen kritisch-politischen Diskursen, sind aber nicht in solche aktiv eingebunden. Sie werden bewusst nicht durch eine vorab gesetzte kritische und/oder postkoloniale Theoriebildung gelenkt. Jene Distanzierung gegenüber präskriptiven Ansätzen ist in besonderem Maße hervorzuheben, weil das längst etablierte und interdisziplinär ausgerichtete Forschungsfeld der Postcolonial Studies die Kolonialgeschichte und deren Auswirkungen in den europäischen Metropolen in der Regel vor dem Hintergrund einer theoretischen Setzung im Sinne des Aufzeigens einer postkolonial-kritischen Theorie verfolgen. Die deutliche Akzentuierung des in der Linguistik seit Langem etablierten und favorisierten deskriptiven Ansatzes für koloniallinguistische Arbeiten liegt mitunter also auch daran, dass man sich vom „kritische[n] Revisionismus der politischen Spielarten“ (Warnke et al. 2016: 10) der Postcolonial Studies dezidiert abgrenzen möchte. Jene eindringliche Betonung dezidiert deskriptiver Zugriffsweisen erfolgt nicht zuletzt

\footnotetext{
55 „Offizielle Bez. des deutschen Staates zwischen 1871 und 1945, also für das Kaiserreich, die Weimarer Republik und für den Staat in der Zeit der Diktatur des Nationalsozialismus, der häufig als Drittes Reich bez. wird“ (Holtmann 2000: 126).
} 
auch aufgrund jüngster Forschungen der Critical Toponymies, deren Interesse sich offenbar auch auf kolonial und postkolonial geprägte Landschaften auszuweiten scheint. ${ }^{56}$ Gerade die etwaige Übernahme eines „Blickwinkel[s] einer postkolonial informierten kritischen Distanz, die aber immer in Gefahr steht, aktuelle Überzeugungen zum Maßstab analytischer Interessen $\mathrm{zu}$ machen“ (Warnke et al. 2016: 18), wäre für den deskriptiv-wissenschaftlichen Charakter der vorliegenden Untersuchung nicht nur problematisch, sondern könnte die übergeordnete Leitfrage weder bearbeiten noch beantworten. Stattdessen müssen etablierte Vorgehensweisen der deskriptiv und empirisch arbeitenden Linguistik für die vorliegende Studie zur Anwendung kommen. Alle Arbeitsschritte erfolgen deskriptiv, kriterienbasiert und datenbasiert: In einem ersten Schritt wird der sprachhistorische Untersuchungsgegenstand im systematisch-ortsübergreifenden Zugriff erhoben. Das zusammengestellte Inventar ist sodann strukturiert mit Annotationen zu versehen, um es in einem weiteren Schritt für daran anschließende Schlussfolgerungen im Sinne etwaiger (sprachhistorischer) Generalisierungen auszuwerten.

\subsubsection{Abgrenzungen}

Die vorliegende kolonialtoponomastische Arbeit, die im sprachhistorischen, ortsübergreifenden und deskriptiven Zugriff erstmalig das koloniale Namenprojekt im Raum der Kolonisatoren identifiziert, inventarisiert und analysiert, unterscheidet sich nicht nur von Studien der Postcolonial Studies und den Critical Toponymies. Hinsichtlich der zu beantwortenden Fragen und des methodischen Untersuchungsinstrumentariums weicht sie auch deutlich von aktuellen Ansätzen gesellschaftspolitischer Akteurinnen und Akteure in Deutschland ab. Diese widmen sich auf Grundlage der von ihnen eingeforderten postkolonialen Erinnerungskulturen unter anderem „Spuren der Kolonialvergangenheit“"57. Solche

56 Vom 18.-20. September 2017 fand ein internationales Symposion in Windhoek, Namibia, unter dem Titel Critical Toponymy: Place names in political, historical and commercial landscapes statt (http://www.igu-icatoponymy.org/2017-international-symposium-on-placenames-windhoek-namibia-18-20-september-2017/, Abruf am 28/06/2019). Mehrere Tagungsvorträge thematisierten Toponyme, die in unmittelbaren und/oder mittelbaren kolonialen und postkolonialen Kontexten verortet werden können. Man vgl. dazu bspw. die Beiträge von Kahari ("A critical study of Zimbabwe's toponymy of place names in historical, political and commercial landscapes") und Mangena ("From Rhodes Memorial Preparatory School to Matopos Primary School: memory, history and symbolic resistance in Zimbabwe”).

57 http://freedom-roads.de/frrd/willkom.htm, Abruf am 28/06/2019. 
postkolonial.de-Gruppierungen widmen sich einzelortsbezogen ${ }^{58}$ oder in Ansätzen ortsübergreifend auch Straßennamen, verfolgen mit einem überwiegend eklektizistischen, lokal und appellativ intendierten Ansatz aber ganz andere Ziele. So fordern sie die

Umbenennung der Straßen [...], die Kolonialverbrecher würdigen. Straßennamen, die das ehemalige deutsche Kolonialreich vergegenwärtigen oder an Kolonialwaren oder koloniale Infrastrukturen erinnern, sollen mit einem Zusatzschild unter dem Straßennamen historisch kontextualisiert werden. ${ }^{59}$

Dabei liegt ihr Fokus auf rezenten Straßennamen, indem sie diese, verbunden mit Umbenennungsforderungen oder deren Kontextualisierung in der unmittelbaren Raumdeskription, aus gegenwartssprachlicher Perspektive und in kritischer Intention zur Diskussion stellen. Dabei ist ein breites Spektrum möglicher Abgrenzungen und Zuordnungen zu erkennen, die in einem früheren Beitrag (vgl. Schulz und Ebert 2017: 167-169) beschrieben wurden.

Sowohl die methodischen Vorgehensweisen als auch die Inventare der postkolonial.de-Gruppierungen unterscheiden sich von dem zusammengestellten sprachhistorischen Nameninventar der vorliegenden Studie. Gesellschaftspolitische Akteurinnen und Akteure gehen nicht ex ante vor, die Aufdeckung der historischen Benennungsmotivik einzelner Namen im Zuge der Einschreibung spielt eine höchstens untergeordnete Rolle. Sie zielen auch nicht auf die systematische Zusammenstellung eines Inventars auf Basis eines groß angelegten Ortskorpus ab, um dieses anschließend vor dem Hintergrund der vorliegenden sprachlichen Strukturen linguistisch zu kommentieren. Die von derartigen Akteuren eingenommene aktuelle Sicht auf Namen ist eindeutig als ex post zu beschreiben. Dabei werden maßgeblich gegenwartssprachliche semantische Bezüge hergestellt, die einen argumentativen Ausgangspunkt gesellschaftspolitisch nötiger Veränderungen wie etwa Straßenumbenennungen zum Ziel haben. Diese sollen weder evaluierend kritisiert oder bestätigt werden. Nichtsdestotrotz bleibt festzuhalten, dass sie ganz andere Absichten verfolgen, die sich von der empirischen Sprachwissenschaft und ihrer datenbasierten Vorgehensweisen unterscheiden.

58 Bspw. für Dortmund (http://www.dortmund-postkolonial.de/?p=1819, Abruf am 28/06/2019), Freiburg (http://www.freiburg-postkolonial.de/, Abruf am 28/06/2019), Hamburg (http://www. afrika-hamburg.de/, Abruf am 28/06/2019), Köln (http://www.kopfwelten. org/kp/, Abruf am 28/06/2019) und München (http://muc.postkolonial.net/, Abruf am 28/06/2019).

59 http://www.freedom-roads.de/frrd/staedte.htm, Abruf am 28/06/2019. 


\subsection{Ortskorpus}

Die systematische Auswahl und Zusammenstellung des kriterienbasierten historisch angelegten Ortskorpus richtete sich nach historischen Bevölkerungszahlen und umfasst Groß- und Mittelstädte: „Noch heute gültig ist die Definition des Statistiker-Kongresses von 1887, nach der [...] Mittelstädte [...] 20.000 100.000 Einwohner aufweisen “ (Flacke 2004: 27). Großstädte sind demnach alle Orte mit über 100.000 Einwohnerinnen und Einwohnern. Die Zusammentragung aller Groß- und Mittelstädte erfolgte mithilfe der von 1881 bis 1943 jährlich vom Statistischen Reichsamt herausgegebenen Zeitschrift Statistische Jahrbücher für das Deutsche Reich. ${ }^{60}$ Für die Zusammenstellung möglichst aller innerhalb dieses Zeitraums relevanten Groß- und Mittelstädte wurde ein diachroner Zugang gewählt, indem in vier Zeitschnitten $(1880,1900,1925,1940)$ alle Ortspunkte mit mehr als 20.000 Einwohnerinnen und Einwohnern aus den jeweiligen veröffentlichten Zeitschriftenbänden zusammengetragen wurden. ${ }^{61}$ Die Erhebung von Groß- und Mittelstädten im historischen Längsschnitt ermöglichte, dass auch solche Ortspunkte mit ins Korpus aufgenommen wurden, die nach dem Ersten oder auch nach dem Zweiten Weltkrieg nicht mehr dazugehörten. Schon nach dem Ersten Weltkrieg gilt das bspw. für die durch die Bestimmungen des Versailler Vertrags an Frankreich ${ }^{62}$, Polen ${ }^{63}$ und der neu gegründeten Tschechoslowakei ${ }^{64}$ abgetretenen Orte. Das gilt auch für alle Groß- und Mittelstädte innerhalb derjenigen Gebiete, die 1945 im Zuge des Potsdamer Abkommens und der dort erfolgten geographischen Neuordnung Deutschlands abgetreten wurden. In dem erstellten Ortskorpus sind bspw. über $100 \mathrm{Groß-}$ und Mittelstädte vertreten, die bis $1945 \mathrm{zu}$ den preußischen Provinzen gehörten. ${ }^{65}$ Die Zusammenstellung dieses in ausgewählten Zeitschnitten angelegten Ortskorpus ermöglichte darüber hinaus, dass auch Städte der 1938 bzw. 1939 vom Nationalsozialistischen Deutschen Reich als Reichsgaue besetzten Gebiete in die Untersuchung mit einbezogen werden konnten. Die betreffenden Städte

60 Alle Bände liegen in digitalisierter Form vor und sind online über das von der Deutschen Forschungsgemeinschaft (DFG) geförderte deutsche digitale Zeitschriftenarchiv zugänglich (https://www.digizeitschriften.de/startseite/, Abruf am 28/06/2019).

61 Volkszählung 1880: 115 Groß- und Mittelstädte. 1900: 206 Groß- und Mittelstädte. 1925: 243 Groß- und Mittelstädte. 1940: 375 Groß- und Mittelstädte.

62 Bspw. Kolmar [Colmar], Mühlhausen [Mulhouse] und Straßburg [Strasbourg].

63 Bspw. Dirschau [Tczew] und Königshütte [Chorzów].

64 Bspw. Eger [Cheb].

65 Bspw. Allenstein [Olsztyn], Breslau [Wrocław], Königsberg i. Pr. bzw. Königsberg (Pr.) [Kaliningrad], Marienburg [Malbork], Stettin [Szczecin]. 
werden unter „Gebietseinteilung und Bevölkerung“ (Statistisches Jahrbuch 1940: 7-41) unter Angabe der jeweiligen Reichsgaue tabellarisch aufgelistet. Um herauszufinden, inwieweit koloniale Benennungspraktiken auch in den jeweiligen Reichsgauen Verwendung fanden, wurden die relevanten Groß- und Mittelstädte ins Ortskorpus aufgenommen.

Nach der Tilgung von Dubletten, die in zwei und mehreren Volkszählungen als Groß- bzw. Mittelstädte aufgelistet sind, konnte ein umfangreiches nach historischen Kriterien angelegtes Ortskorpus zusammengestellt werden, das 430 Groß- und Mittelstädte des Deutschen Reichs umfasst. Darunter finden sich teilweise auch Zuschnitte der heutigen Städte mehrfach: Für Wuppertal sind das bspw. Barmen, Elberfeld und Vohwinkel, die in den Volkszählungen von 1880, 1900 und/oder 1925 als eigene Groß- bzw. Mittelstädte auftauchen, aber noch innerhalb des Untersuchungszeitraums eingemeindet wurden. ${ }^{66}$ Im Appendix 2 wird das erstellte Ortskorpus daher ohne die bis 1945 erfolgten Eingemeindungen dargelegt. Es umfasst über 370 Groß- und Mittelstädte. Der gewählte diachrone Zugang der ortsübergreifenden Zusammenstellung eines kolonial motivierten Inventars ermöglicht nicht nur linguistische Analysen des ortsübergreifenden Gesamtinventars, sondern schafft zugleich die Voraussetzungen, strukturelle und diskursfunktionale Vergleiche von Teilinventaren innerhalb der drei unterschiedlichen gesellschaftspolitischen Benennungszeiträume (Deutsches Kaiserreich, Weimarer Republik, NS-Zeit) vorzunehmen.

\subsection{Quellen}

Der deskriptive Zugriff aus der sprachgebrauchsgeschichtlichen Perspektive ex ante erforderte eine erstmalige Identifizierung etwaiger kolonial motivierter Straßennamenvergabepraktiken im Beobachtungszeitraum von 1884 bis 1945 auf Basis des erstellen Ortskorpus, das sowohl in zeitlicher als auch in räumlicher Perspektive den Raum des Deutschen Reichs systematisch erfasst. In der Praxis wurden alle über 400 Groß- und Mittelstädte hinsichtlich etwaiger kolonial motivierter Straßenbenennungen untersucht. Jenes zeitintensive Unterfangen orientierte sich an der unmittelbaren inhaltlichen Zuschreibung einer historischen Benennung zum zeitgenössischen Kolonialdiskurs, d.h. es wurden nur diejenigen sekundären Straßennamen inventarisiert, die sich nachweislich zum

66 Wuppertal taucht bspw. erst im weiteren Schnitt von 1940 auf, weil sie als kreisfreie Stadt erst 1929 durch Vereinigung, u. a. durch die zuvor genannten Städte, gegründet wurde (http:// www.archive.nrw.de/kommunalarchive/kommunalarchive_u-z/w/Wuppertal/wir_ueber_uns/ index.php, Abruf am 28/06/2019). 
historischen Zeitpunkt ihrer administrativen Verfügung auch unmittelbar auf Kolonialismus und koloniale Themen beziehen sollten. Die Überprüfung der Erstglieder von Straßennamen auf etwaige Artikel in den noch zur faktischen Kolonialzeit verfassten Koloniallexika (vgl. Kausch 1903, Schnee 1920) gibt erste Hinweise darauf, ob die sprachlichen Einheiten innerhalb des historischen Kolonialdiskurses zu verorten sein könnten. Der Nachweis einer unmittelbaren kolonialen Benennungsmotivik kann aber nur im tokenbezogenen Zugriff durch weitere quellenbezogene Verfahren realisiert werden.

\subsubsection{Koloniallexika}

Die zwei enzyklopädisch aufgebauten umfangreichen Koloniallexika von Kausch (1903) und Schnee (1920) sind noch während der faktischen Kolonialepoche in der Zeit des Kaiserreichs verfasst worden. ${ }^{67}$ Für diese Arbeit sind die historischen Wörterbuchartikel relevant, die in den jeweiligen Lexika zusammengetragen wurden und mit denen zügig nachgeprüft werden kann, inwieweit die linksköpfigen Erstglieder der zweigliedrigen Konstruktionen sekundärer Straßennamen auch durch entsprechende Einträge in den jeweiligen Publikationen vertreten sind. Zur Person Carl Peters, die in zeitgenössischen Quellen als „bedeutendste[r] Kolonialpolitiker Deutschlands“ (Schorn 1920: 101) gefeiert wurde, findet sich zur Biographie und zu seinem kolonialpolitischen Wirken bspw. folgender Eintrag in Schnee (1920 III: 40):

Peters, Carl, Reichskommissar a. D., Dr. phil. [...] 1881/83 hielt er sich in London auf, kehrte dann nach Berlin zurück und begann sich bald ganz der praktischen Kolonialpolitik zuzuwenden. Er gründete hierzu 1884 die "Gesellschaft für deutsche Kolonisation” [...] Es gelang hier durch mehrere Verträge die Landschaften Usagara, Nguru, Useguha und Ukami für die Kolonisationsgesellschaft zu erwerben und hierfür einen Kaiserlichen Schutzbrief zu erhalten (27. Febr. 1885). P. ist somit als der Begründer des heutigen Ostafrika anzusehen [...].

Durch die Recherche in den historischen Koloniallexika kann schnell festgestellt werden, ob die relevanten linksköpfigen Erstglieder im zeitgenössischen kolonialen Diskurs tatsächlich als relevant und unmittelbar auf die Kolonien

67 Das von H. Schnee herausgegebene Koloniallexikon wurde erst 1920 publiziert, wurde aber bereits 1914 und damit noch in der Zeit der de facto-Kolonialzeit fertiggestellt. Es liegt in digitaler Form vor. Man vgl. dazu das Forschungsprojekt Der Bildbestand der Deutschen Kolonialgesellschaft, Universitätsbibliothek Frankfurt am Main (http://www.ub.bildarchiv-dkg.uni-frankfurt.de/, Abruf am 28/06/2019). 
und Schutzgebiete bezogen verstanden wurden. Außerdem wurde die Digitale Sammlung Deutscher Kolonialismus (DSDK) ${ }^{68}$ genutzt, die „historische, zeitgebundene Sammlungspraktiken selbst zu rekonstruieren und damit ein Archiv ex ante [kursiv im Original] abzubilden“ (Warnke und Schmidt-Brücken 2017: 947) versucht. Sowohl die Koloniallexika als auch die DSDK stellen jedoch - insbesondere bei Familiennamen von Personen - kein ausreichendes Kriterium für die Inventarisierung eines SN-Tokens zum kolonial motivierten Gesamtkorpus dar: Die Identifizierungsaufgabe ist nur dann erfolgreich, wenn nachgewiesen werden kann, dass sich das SN-Token nicht nur ausdrucksseitig, sondern auch inhaltsseitig auf Kolonialakteure beziehen sollte. Die Überprüfung des unmittelbaren kolonialen Gehalts einer historischen Benennung bezüglich der intendierten Motivik kann nur durch weitere quellenbezogene Verfahren realisiert werden. Dies gilt insbesondere dann, wenn Straßennamen mit ausdrucksseitig identischen Erstgliedern vorliegen, die sodann auf eine eindeutige Aussage in Bezug auf das Inventar kolonial motivierter Namen zu überprüfen sind. ${ }^{69}$ Die fundierte Zuschreibung eines SN-Tokens zum kolonial motivierten Namenkorpus basiert sodann auf inhaltsseitigen, historisch nachweisbaren Kriterien. Sie ist nur auf Basis diverser Quellen und Quellengattungen möglich, die sicherstellen, dass die jeweiligen sprachlichen Einheiten zum Benennungszeitpunkt von der jeweiligen städtischen Administration tatsächlich im unmittelbaren Kontext des Kolonialismus verortet wurden. Das ist bspw. der Fall, wenn ein städtisches Gremium beschließt, dass ein Straßenzug, der „parallel mit der Halleschen Chaussee geht [...] den Namen Petersstraße (nach dem Kolonialpionier Dr. Carl Peters) [erhält]“ (Mitteilung des Polizeipräsidenten von Merseburg 15.07.1937).

Als zielführend erweist sich ein Inventarisierungsverfahren additiver Informationsgewinnung zur systematischen Sichtung aller möglichen und verfügbaren Quellen. Für die konsistente Ermittlung kommen vor allem historische Quellengattungen in Frage. Daneben werden aktuelle (Informations- und Forschungs-)Publikationen, insbesondere Straßennamenlexika, genutzt. Die Sich-

68 http://brema.suub.uni-bremen.de/dsdk, Abruf am 28/06/2019.

69 Schulz und Ebert (2017: 167) zeigen die dafür erforderlichen Abgrenzungsschritte anhand des SN-Tokens Petersstraße für den Ortspunkt Leipzig auf. „Die Überprüfung historischer Adressbücher macht deutlich, dass der Straßenname [...] benennungsmotivisch [...] auf einen Heiligennamen [verweist]“. Bei der Abgrenzung der in Leipzig verfügten Petersstraße handelt es sich um einen einzelortsbezogenen Befund auf der Token-Ebene: „Für die Aufdeckung der Situation in anderen Städten sind weitere Einzelanalysen erforderlich, an deren Ende der Befund bezüglich Petersstraße natürlich ganz anders ausfallen kann. So ist dieser Straßenname etwa für den Ortspunkt Düsseldorf klar als kolonial intendierter Straßenname identifizierbar“ (Schulz und Ebert 2017: 167, Fn. 14). 
tung der historischen Quellengattungen umfasst neben administrativen (Verfügungsakten) und nicht-administrativen (z. B. Zeitungsartikel) Texten insbesondere zeitgenössische Einwohner- bzw. Adressbücher sowie Stadtpläne, die sich als besonders hilfreich erweisen. Zur Schließung von Lücken wurde zudem der Kontakt zu lokalen Archiven hergestellt, um relevante historische Straßenbenennungsakten zu erfragen. Die für den konsistenten Ermittlungsschritt maßgeblichen Quellengattungen werden in den nun folgenden Unterkapiteln vorgestellt.

\subsubsection{Straßennamenlexika}

Bei Straßennamenlexika handelt es sich um einzelortsbezogene Nachschlagewerke, die nicht sprachwissenschaftlich ausgerichtet sind. Vielmehr handelt es sich um Publikationen, die vorrangig in archivarischen, administrativen, historischen oder informierenden Kontexten stehen und sich Straßennamen (und ihrer historischen Erklärung) einzelortsbezogen als Teil der Lokalgeschichte widmen; in der Regel führen sie den zum Zeitpunkt der jeweiligen Veröffentlichung rezent vorliegenden Straßennamenbestand für einen bestimmten Ort auf. Die in derartigen Schriften alphabetisch angelegten Straßennamenartikel werden häufig mit weiteren Informationen versehen, bspw. mit der Lage des jeweiligen Straßenzugs, dem Benennungsjahr der Namenverfügung u. dgl., die maßgeblich auf Quellenbestände ortsansässiger Stadtarchive, Bestände von Landesbibliotheken oder auf Informationen stadtgeschichtlicher Projekte beruhen, die für derartige Straßennamenlexika entsprechend zusammengetragen und ausgewertet werden. Straßennamen mit kommemorativer Funktion werden in solchen Nachschlagewerken häufig administrativen Benennungsmotiviken angeführt. ${ }^{70}$ Neben der Darlegung des rezenten Namenmaterials widmen sich einige Straßennamenlexika auch diachron vergleichenden Darstellungen, mit dem Ergebnis, dass auch bereits getilgte und umbenannte historische Straßennamen erläutert werden. ${ }^{71}$

70 Bspw. wird im Straßennamenlexikon der bayerischen Hauptstadt München angegeben, dass die Benennung Danziger Straße 1923 verfügt wurde und auf „Danzig, der Stadt an der Ostsee, frühere deutsche Hansestadt, heute poln. Gdansk [...]“ referiert. Die 1932 verfügte Benennung Damaschkestraße bezieht sich auf „Adolf Damaschke (1865-1935), Sozialpolitiker und Nationalökonom; Vorsitzender des ,Bundes deutscher Bodenreformer “ (Dollinger 2007: 59).

71 Bspw. findet sich im Straßennamenlexikon von Kiel ein eigener Eintrag zum 1947 getilgten und umbenannten SN-Token Scheerstraße (in Mecklenburger Straße), das in den 1930er Jahren nach „Reinhard Scheer (20.9.1863-26.11.1928), Admiral, 1916-18 Chef der Hochseeflotte“ (Hilscher 2015: 163) verfügt wurde. 
Der Nutzen dieser Straßennamenlexika zur Informationsgewinnung für die vorliegende Arbeit hängt nicht nur von der individuell erarbeiteten Informationstiefe der einzelnen Werke ab. Auch strukturell sind einer historisch-onomastischen Informationsgewinnung Grenzen gesetzt. Sänger (2006: 48) hat bereits in einem anderen Kontext darauf hingewiesen, dass die in solchen Publikationen erarbeiteten Datensätze, die dafür gewählte Methodik und mitunter die wissenschaftliche Qualität der jeweiligen Einträge stark von der Autorinnenund Autorenschaft abhängig sind. Dass sich solche Namenbücher zur Beantwortung onomastischer Fragestellungen nur in bedingtem Ausmaß eignen, wurde auch von Seiten der Forschungsstelle zu den Kölner Straßennamen herausgestellt:

Die [...] Straßennamenlexika genügen zumeist nicht einmal enzyklopädischen Anforderungen [...]: Häufig finden sich allein Erläuterungen zur Bedeutung des Namens, nicht aber das Benennungsjahr und die früheren Bezeichnungen der Straße, welche Angaben doch erst den Symptomwert der Bedeutung valide machen. (Bering et al. 1999: 140, Fn. 29)

Für das in dieser Arbeit erstellte Ortskorpus liegen nur für weniger als 40 Großund Mittelstädte derartige Namenlexika vor. Die wissenschaftliche Qualität der in den entsprechenden Publikationen erarbeiteten Datensätze zeigt für die Fragestellung der vorliegenden Studie eine ganz unterschiedliche Erschließungstiefe. Dörfler (2006: 45) weist bereits darauf hin, dass jene lokalspezifischen Publikationen aufgrund der „etymologischen Methode, der fehlenden intersubjektiven Überprüfbarkeit ihrer Ergebnisse und ihrer nicht immer haltbaren Schlüsse tatsächlich nur auf der anekdotisch-heimatkundlichen und nicht auf der wissenschaftlichen Ebene anzusiedeln [sind]“. Zweckmäßig waren vor allem diejenigen Lexika, die den lokalen Straßennamenbestand in diachronen Zuschnitten darlegen, historische Benennungen für den Untersuchungszeitraum dieser Arbeit mit entsprechenden Namenartikeln berücksichtigen und damit die Kategorie der inhaltlichen Zuschreibung zu etwaigen kolonialen Themen - unter Angabe des hierfür verwendeten Quellenmaterials - belegen. Dazu gehört bspw. das Kieler Straßenlexikon: Für die 1939 durch den örtlichen Polizeipräsidenten verfügten SN-Token Carl-Peters-Straße, Lettow-Vorbeck-Straße, Lüderitzstraße, Nachtigalstraße, Wissmannstraße und Woermannstraße für aneinander liegende Straßenzüge in Neumühlendorf-Dietrichsdorf sind jeweils eigene Namenartikel aufgeführt, die mit folgenden Metadaten versehen sind: 


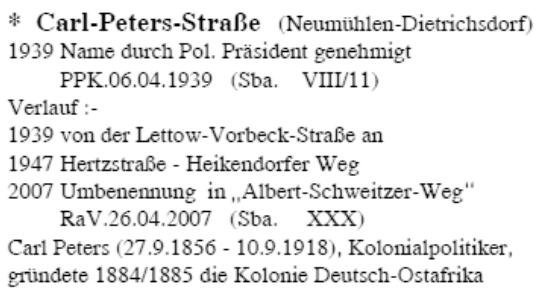

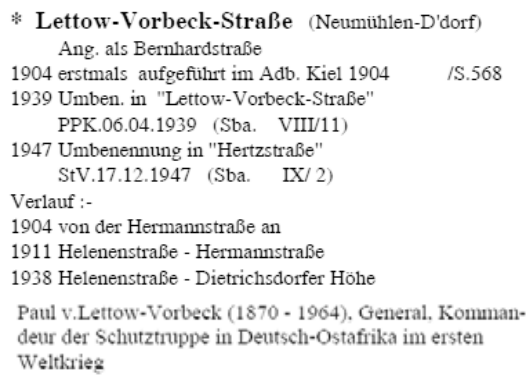

Abb. 2: Einträge Carl-Peters-Straße und Lettow-Vorbeck-Straße in Hilscher $(2015: 37,120)$.

Alle Straßenbenennungen referieren zum Zeitpunkt ihrer Namenvergabe am Ende der 1930er Jahre in kommemorativer Intention auf Kolonialakteure aus der Kaiserzeit. $^{72}$ Solche in diachron-vergleichender Perspektive erarbeiteten Namenlexika, die auch bereits umbenannte kolonial motivierte Namen mit entsprechenden Informationen zu den historischen Benennungsumständen und den dafür genutzten Quellen nachweisen, können für die umfangreiche Inventarisierungsaufgabe hinzugezogen werden. Neben der Sichtung derartiger aktueller Informations- und Forschungsliteratur war aber insbesondere die Auswertung historischer Quellen und Quellengattungen erforderlich, die nun dargelegt werden.

\subsubsection{Straßenbenennungsakten}

Administrative Akten zu historischen Straßenbenennungen geben detailgenaue Auskünfte über Benennungsjahr, -motiviken und -umstände und sind in ortsansässigen Stadtarchiven einsehbar. Allerdings ist die Aktenüberlieferung für eine Vielzahl an Groß- und Mittelstädten des Ortskorpus bis in die erste Hälfte des 20. Jahrhunderts kriegsbedingt als rudimentär zu beschreiben. ${ }^{73}$ Für einen Groß-

72 Die Benennungsmotiviken am Ende der jeweiligen Einträge stammen aus historischen Adressbüchern (vgl. Hilscher 2015: 6). Man vgl. dazu bspw. den Eintrag zur Lettow-VorbeckStraße im Kieler Adressbuch (1940 II: 160): „Paul von Lettow-Vorbeck, Generalmajor, erfolgreicher Verteidiger der deutschen Kolonien in Afrika im Weltkrieg“.

73 In diesem Zusammenhang sollen nur einige Auszüge von Mailkorrespondenzen dargelegt werden, die ich auf Nachfrage von Seiten der Mitarbeiterinnen und Mitarbeitern der ortsansässigen Stadtarchive erhalten habe: Darmstadt: „Durch die fast vollständige Aktenvernichtung im September 1944 haben sich überhaupt keine Vorkriegsakten zum Thema Straßenbenennung erhalten“; Dessau-Roßlau: „Akten hierzu sind leider nicht erhalten geblieben“; Eilenburg: „die Quellenlage für die relevanten Zeiträume zur Eilenburger Ortsgeschichte [ist] sehr 
teil der Städte liegen amtliche Straßenverzeichnisse für den die vorliegende Arbeit betreffenden Untersuchungszeitraum, oftmals aufgrund vollständiger Aktenvernichtung gegen Ende des Zweiten Weltkrieges, nicht vor. Im Rahmen des Forschungsvorhabens war es nur möglich, telefonisch oder per Brief- bzw. Mailverkehr mit den lokalen Archiven zu kommunizieren. Eine flächendeckende persönlich vorzunehmende Autopsie in den Archiven der über 400 Ortspunkte ist völlig unrealistisch. Selbst die nicht ausschließbare Chance, bei umfangreichen eigenen Archivstudien punktuell auf weitere relevante Benennungsakten zu stoßen, kann ein solches Vorhaben nicht rechtfertigen. Auffällig ist, dass vor dem Hintergrund des derzeitigen öffentlichen Interesses an Umbenennung vereinzelter rezenter Straßennamen, deren historische Benennungen auf koloniale Themen zurückzuführen sind, die historischen Benennungsumstände wieder verstärkt thematisiert und durch Archive aufgearbeitet bzw. online bereitgestellt werden. Dies erfolgt derzeit aber nur einzelortsbezogen, so bspw. für Kaiserslautern. ${ }^{74}$

\subsubsection{Adressbücher}

Historische Adress- bzw. Einwohnerbücher listen (neben alphabetischen Einwohner- und Firmenverzeichnissen sowie Werbeanzeigen) auch Straßenverzeichnisse auf, in denen der jeweilige Namenbestand vom Anfang des angegebenen Jahres wiedergegeben wurde. Eine quellenkritische Einschätzung der Bremer Adressbücher, die sich auf die zwischen 1794 und 1985 erstellten Einwohnerbücher bezieht, liegt von Adolfmeister (1989: 9-10) vor:

kompliziert. Neben komplett fehlenden örtlichen Akten, Meldeunterlagen, Personenstandsakten haben sich nicht einmal die Tageszeitungen dieser Zeit überliefert“; Forst: „Zeitgenössische Akten zum Thema oder ortsgeschichtliche Arbeiten dazu konnte ich in unseren Beständen nicht ermitteln“; Gotha: „Die Akten zu Straßen(um)benennungen sind leider im relevanten Zeitraum nicht mehr überliefert“; Kleve: „Die Aktenüberlieferung der Stadt Kleve für den für Sie interessanten Zeitraum ist - kriegsbedingt - sehr schlecht. Amtliche Straßenverzeichnisse fehlen“; Minden: „Und die einschlägigen Akten des Vermessungsamtes, aus denen vielleicht Näheres ersichtlich wäre, sind nicht überliefert“; Nordhausen: „Leider sind durch kriegsbedingte Zerstörung 1945 umfangreiche Aktenbestände der Stadtverwaltung aus preußischdeutscher bzw. NS-Zeit verlorengegangen, so dass der evtl. Hintergrund und Beschlüsse zu Straßenbenennungen vor 1945 [...] schlecht zu erforschen sind“.

74 Man vgl. dazu die Bürgerinformation vom 08.01.2015 der Stadt Kaiserslautern, die die historische Straßenbenennung Karl-Peters-Straße, allerdings vor dem Hintergrund der Umbenennung, erläutert (https://ris.kaiserslautern.de/buergerinfo/vo0050.php?_kvonr=3159, Abruf am 28/06/2019). 
Es ist schwierig festzustellen, wie die Adreßbuchverlage sich in älterer Zeit die nötigen Informationen beschafften. Zum großen Teil waren sie wohl auf eigene Erhebungen angewiesen, dazu traten Auskünfte der Behörden. [...] Da sich die Adreßbücher im täglichen Gebrauch zu bewähren hatten und offenbar nach den ersten drei Jahrzehnten auch bewährt haben, kann ihre Zuverlässigkeit im Allgemeinen hoch eingeschätzt werden. Andererseits ist mit Irrtümern, Druckfehlern, Unvollständigkeit im Einzelnen sowie mit Verzögerungen bei der Aufnahme, Änderung und Löschung der Daten stets zu rechnen.

Für die sprachwissenschaftlichen Fragestellungen der vorliegenden Studie und der systematischen Zusammenstellung eines Inventars kolonialer Straßenbenennungen bis 1945 erwiesen sich die in den historischen Adressbüchern aufgeführten Straßenverzeichnisse als besonders hilfreich: Neben der systematischen Überprüfung der Namenbestände in diachronen Zeitschnitten stellen sie häufig weitere Informationen zur Lage sowie Nachweise $\mathrm{zu}$ Benennungsmotiviken sekundärer Straßennamen bereit. ${ }^{75}$ Die Motiviken zeigen damit die für die $\mathrm{Zu}$ ordnung historischer Benennungen $\mathrm{zu}$ dem in dieser Arbeit $\mathrm{zu}$ untersuchenden kolonialen Straßennamen notwendigen inhaltsseitigen Kriterien auf, die durch die administrative Aktenüberlieferung allenfalls punktuell abgedeckt wird. Am Beispiel des SN-Tokens Lettow-Vorbeck-Straße, das in nationalsozialistischer Zeit mit weiteren kolonial motivierten Benennungen als Kolonialcluster in das neu aufzuschließende Viertel Braunschweig-Querum verfügt wurde, soll dargelegt werden, welche Informationen aus den Einträgen in Straßenverzeichnissen gewonnen werden können (Abb. 3):

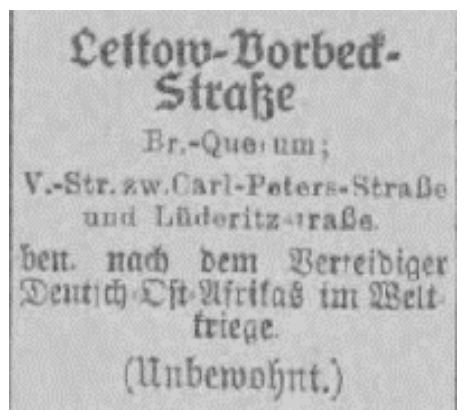

[SN-Token]: Lettow-Vorbeck-Straße

[Verortung]: Braunschweig-Querum;

Verbindungs-Straße zwischen Carl-Peters-Straße und Lüderitzstraße.

[Motivik]: Benannt nach dem Verteidiger Deutsch-Ost-Afrikas im Weltkriege.

[Einwohner]: Unbewohnt.

Abb. 3: Adressbuch Braunschweig (1940 IV: 207).

75 Bspw. findet sich im Straßenverzeichnis für München (Adressbuch 1923 II: 368) für den Kaiser Ludwigsplatz folgende Bezeichnungsmotivik: „Kaiser Ludwig der Bayer, geb. 1. April 1282, gestorben [...] 1347.“); im Straßenverzeichnis des Adressbuches der Stadt Kiel (1940 II: 86) wird bspw. für den sekundären Straßennamen Hardenbergstraße angegeben, dass sich dieser auf den „Fürst Karl August v. Hardenberg, ehem. Preußischer Minister [...]“ bezieht. 
Neben der Verortung und dem impliziten Hinweis, dass es sich um eine Erstbenennung eines neu angelegten Straßenzugs handelt, ist die Benennungsmotivik hervorzuheben: Sie zeigt auf, dass die Namenverfügung Lettow-Vorbeck-Straße auf „den Verteidiger Deutsch-Ost-Afrikas im Weltkriege“ referiert. In dem für die städtische Öffentlichkeit zugänglichen Text werden damit historische Zuschreibungen vorgenommen, die die genuin koloniale Intention des verfügten SN-Tokens darlegen. Der Vergleich mit den in administrativen Verordnungen dargelegten Motiviken zeigt, dass es sich bei den in Adressbüchern vorgenommenen Zuschreibungen oftmals um Extrakte administrativer, nicht für die Öffentlichkeit zugänglicher umfangreicherer Texte handelt:

Lettow-Vorbeck-Straße (geb. 20.03.1870). 1913 Kommandeur der Schutztruppe in DeutschOst-Afrika. Gegen eine Übermacht von Feinden verteidigte Lettow-Vorbeck die deutsche Kolonie in Ostafrika und war am Friedensschluß noch nicht besiegt. (Zusammenfassung der Beigeordnetensitzung vom 17.11.1939 zur Straßennamensgebung in Braunschweig)

Daneben finden sich auch explizitere Verweise als in den administrativen Verordnungen, was am Beispiel der Namenverfügung Carl-Peters-Straße in Braunschweig-Querum gezeigt werden kann:

Carl-Peters-Straße: Der Afrikaforscher Dr. Carl Peters lebte in den Jahren von 1856 bis 1918. Er gründete in den 80er Jahren die Deutsch-Ostafrikanische Gesellschaft. (Zusammenfassung der Beigeordnetensitzung vom 17.11.1939 zur Straßennamensgebung in Braunschweig)

Carl-Peters-Straße [...] ben. [d. i. benannt] nach dem Kolonialpionier Dr. Carl Peters. (Adressbuch Braunschweig 1940 III: 76)

Die im historischen Adressbuch angegebene Motivik zeigt, dass eine ausdrückliche Zuschreibung der Person Carl Peters als Kolonialpionier erfolgte, auf den die Namenverfügung referieren sollte. Neben Extrakten und expliziteren Verweisen für Personennamen sind auch Abschriebe aus administrativen Verordnungen festzustellen, die weitestgehend den administrativen Benennungsmotiviken entsprechen:

Windhuker Straße. Hauptstadt unserer Kolonie Deutsch-Südwestafrika. (Zusammenfassung der Beigeordnetensitzung vom 17.11.1939 zur Straßennamensgebung in Braunschweig)

Windhuker Straße [...] ben. nach der Hauptstadt der deutschen Kolonie Deutsch-SüdwestAfrika. (Adressbuch Braunschweig 1940 III: 319)

Zeitgenössisch ist von einer vielfältigen Rezeption jener Quellengattung auszugehen, in denen nicht nur der Straßennamenbestand, sondern auch historische Zuschreibungen der jeweiligen Benennungen für die städtische Öffentlichkeit dargelegt wurden. 
Die in Straßenverzeichnissen der Adressbücher dargelegten Motiviken sind insbesondere bei ausdrucksseitig übereinstimmenden Erstgliedern hinzuzuziehen, bei denen im Einzelfall zu überprüfen ist, ob die historische Verfügung seitens der Administration intentional auf Kolonialismus und koloniale Themen verweisen sollte. Dies soll an einem weiteren Beispiel, das keine Familiennamen von Personen betrifft (Kap. 3.3.1), gezeigt werden: Die Straßenübersichtsverzeichnisse für Heilbronn (vgl. Adressbuch 1936 III) bzw. Bochum (vgl. Adressbuch 1924/1925 IV) listen jeweils die Straßenbenennungen Karolinenweg bzw. Karolinenstraße mit dem ausdrucksseitig identischen Erstglied Karolinen auf. Das Koloniallexikon führt zu Karolinen einen eigenen Eintrag auf:

Karolinen [...]. Nachdem der Vertrag vom 30. Juni 1899 die Zustimmung der gesetzgebenden Körperschaften gefunden hatte, wurde der gesamte Archipel durch Allerhöchste Order [...] für das Deutsche Reich in Besitz genommen und durch Allerhöchste Order vom gleichen Tage [...] als ein Bestandteil des Schutzgebiets Deutsch-Neuguinea erklärt. (Schnee 1920 II: 239)

Inwiefern die in Heilbronn und Bochum verfügten sprachlichen Einheiten sich auch auf das kolonisierte Inselgebiet im Pazifischen Ozean beziehen und damit innerhalb des zeitgenössischen Kolonialdiskurses $\mathrm{zu}$ verorten ist, ermöglicht die Überprüfung der sich voneinander unterscheidenden Motiviken:

Karolinenweg 1936 nach den Karolineninseln in der Südsee, die bis 1919 deutsches Schutzgebiet waren. (Adressbuch Heilbronn 1936 III: 78)

Karolinenstraße (zwischen Overdyker und Heidestraße.) (Nach der Zeche Karolinenglück.) (Adressbuch Bochum 1924/25 IV: 102)

Das SN-Token Karolinenweg in Heilbronn referiert eindeutig auf das kolonisierte Inselgebiet in Deutsch-Neuguinea im Pazifischen Ozean, das in der Zeit des Kaiserreichs als Kolonie erworben wurde, und gehört zweifellos zu dem in vorliegender Arbeit erstellten Namenkorpus. Mit dem in Bochum eingeschriebenen SNToken verhält es sich trotz des ausdrucksseitig identischen Erstglieds völlig anders: Der im Bochumer Stadtteil Hamme errichtete Straßenzug führt zum Steinkohlen-Bergwerk Zeche Karolinenglück bzw. „Zeche Ver. [d. i. Vereinigte] Carolinenglück. Diese Schachtanlage war in den 1840er angelegt worden“ (Hermann und Hermann 2003: 144). Das SN-Token Glückaufstraße umgibt die bergbauliche Betriebsstätte ebenfalls (vgl. Stadtplan Bochum 1924/25). Sie referiert auf den älteren, 1870 getilgten Zechennamen Glückauf: „Nach der Lage in der Nähe von bergbaulichen Betriebsstätten“ (Adressbuch Bochum 1924/25 IV: 26). Der Benennung der Karolinenstraße in Bochum kann damit keine damit intendierte koloniale Kommemoration nachgewiesen werden. Das SN-Token wurde nicht zum Na- 
menbestand der vorliegenden Untersuchung aufgenommen. Die Beispiele zeigen nochmals deutlich auf, dass der ortsübergreifende Identifizierungsschritt kolonial motivierter Straßenbenennungen nicht ausschließlich auf Grundlage der Erhebung strukturell festgelegter Muster der jeweiligen Erstglieder erfolgen kann. Die Aufdeckung ausdrucksseitiger Strukturen kommemorativer Benennungen in Bezug auf die Erstglieder der zweigliedrigen Konstruktionen ist für den ersten Zugriff durchaus hilfreich; die jeweiligen SN-Token sind sodann zwingend hinsichtlich ihrer mit der Verfügung intendierten Motivik, also vor dem Hintergrund der inhaltsseitigen Zuschreibung zu Kolonialismus und koloniale Themen, quellenbasiert zu überprüfen.

Adressbücher stellen eine leicht zugängliche historische Quellengattung heraus: Sie sind für den historischen Zeitraum (1884-1945) für eine beträchtliche Anzahl an Groß- und Mittelstädten des erstellten Ortskorpus erhalten geblieben. Darüber hinaus sind sie nicht nur in den jeweiligen hiesigen Stadtarchiven einsehbar; eine beachtliche Anzahl der Einwohnerbücher wird durch regionale $^{76}$ und überregionale ${ }^{77}$ Digitalisierungs- und Erschließungsprojekte online zur Verfügung gestellt. Sie sind zugleich methodisches Werkzeug, mit dem sichergestellt werden kann, dass sich die sprachlichen Einheiten zum historischen Benennungszeitpunkt auch auf spezifisch koloniale Kontexte bezogen haben. Für den gewählten Untersuchungszeitraum sind Einwohnerbücher fast ausnahmslos für alle Städte verfügbar, die ununterbrochen bis 1945 zum Deutschen Reich gehörten. Weitaus problematischer stellt sich die Verfügbarkeit für solche Orte dar, die Ende der 1930er Jahre im Zuge des Münchener Abkommens und des Polenfeldzugs erstmalig annektiert wurden und innerhalb des zu untersuchenden Ortskorpus vertreten sind (Kap. 4).

76 Das Projekt der Sächsischen Landesbibliothek - Staats- und Universitätsbibliothek Dresden (SLUB) und des Dresdner Stadtarchivs digitalisiert historische Adressbücher von Städten in Sachsen. Sächsische Landesbibliothek - Staats- und Universitätsbibliothek Dresden (SLUB), Dresdner Stadtarchiv: Projekt zur Digitalisierung und Erschließung sächsischer Adressbücher (https://adressbuecher.sachsendigital.de/startseite/, Abruf am 28/06/2019).

77 Digitalisierte historische Adressbücher werden für eine Vielzahl an Orten kostenfrei über das Portal DigiBib, der Digitalen Bibliothek im GenWiki, zur Verfügung gestellt: „Die Digitale Bibliothek (kurz: DigiBib) im GenWiki sammelt in erster Linie genealogisch und ortsgeschichtlich relevante Literatur, deren Urheberrechte abgelaufen sind“ (http://wiki-de.genealogy.net/ Kategorie:Adressbuch_in_der_DigiBib, Abruf am 28/06/2019). 


\subsubsection{Stadtpläne}

Die Vorteile von historischen Einwohnerbüchern (Bezeichnungsmotiviken, leichte Zugänglichkeit, digitale Verfügbarkeit) für die konsistente ortsübergreifend-nationale Erstellung eines Inventars kolonialer Straßenbenennungen wurden bereits dargelegt. Daneben stellen Stadtpläne eine weitere wichtige Quellengattung dar: Sie legen usuelle raumsemantische Aspekte offen, die sich für die systematische Erstellung eines Gesamtinventars kolonial motivierter Straßennamen als durchaus gewinnbringend erweisen (vgl. Schulz 2018). Dass Benennungspraktiken von sekundären Straßennamen in thematischer Kohärenz als Cluster von Seiten der Administration befürwortet wurden und werden, wurde bereits in Kap. 2.2.2 dargelegt. Für die Identifizierung kolonial motivierter Namen erweisen sich Clusterbenennungen als durchaus nützlich, die in entsprechenden Stadtplänen offengelegt werden - insbesondere in solchen Fällen, in denen Benennungsmotiviken in historischen Einwohnerbüchern nicht oder nur teilweise aufgeführt sind.

Ein ortsbezogenes Beispiel soll dies verdeutlichen: Für die Bremer Vorstadt sind erstmalig 1919 die drei SN-Token Wissmannstraße, Gerhard-Rohlfs-Straße und Nachtigalstraße festzustellen, deren Benennungsmotiviken unmittelbar auf Kolonialakteure des Kaiserreichs referieren (vgl. Adressbuch Bremen 1919 II: 107, 800, 935). Neben diesen drei SN-Token werden im Straßenverzeichnis des gleichen Jahres auch die SN-Token Leutweinstraße und Leutweinplatz für die Bremer Vorstadt aufgeführt (vgl. Adressbuch Bremen 1919 II: 901), allerdings ohne Angabe von Benennungsmotiviken. Dass es sich bei den beiden SN-Token um Benennungen handelt, die auf den gleichnamigen Gouverneur von DeutschSüdwestafrika ${ }^{78}$ referieren sollten, wird durch die Sichtung entsprechender Stadtpläne (vgl. Stadtplan Bremen 1927, 1938) deutlich. Die angelegten Straßen bzw. Plätze liegen in unmittelbarer Nähe und grenzen an den Straßenzug der Wissmannstraße (siehe Abb. 4).

Die Benennung Kolumbus[straße], deren Straßenzug durch das zeitgleich angelegte thematische Viertel führt, wird bereits im Bremer Adressbuch von 1900 (II: 470) aufgelistet. Eine etwaige koloniale Motivik kann für das SN-Token nicht nachgewiesen werden. ${ }^{79}$ Der Miteinbezug der in Stadtplänen zum Aus-

78 Man vergleiche dazu den entsprechenden Eintrag in Schnee (1920 II: 452): „Leutwein [Fettdruck im Original], Theodor Gotthilf, Ksl. Generalmajor z.D. und früher Gouverneur von Deutsch-Südwestafrika. [...] 1894 Feldzug gegen Hendrik Witboi [...], 1896 gegen Hereros [...] und Kauashottentotten [...], 1904 gegen Hereros (s. Hereroaufstand).“

79 „Columbusstraße [...] Columbus, der Entdecker Amerikas, ein Genuese, geboren 1446, gestorben 1506“ (Adressbuch Bremen 1919 III: 88). 
druck kommenden Raumsemantik lässt zweifelsohne darauf schließen, dass es sich bei Leutweinstraße und Leutweinplatz um kolonial motivierte Benennungen handelt, die mitsamt weiterer zeitgleich erfolgten Benennungen als Kolonialcluster in Walle verfügt wurden.

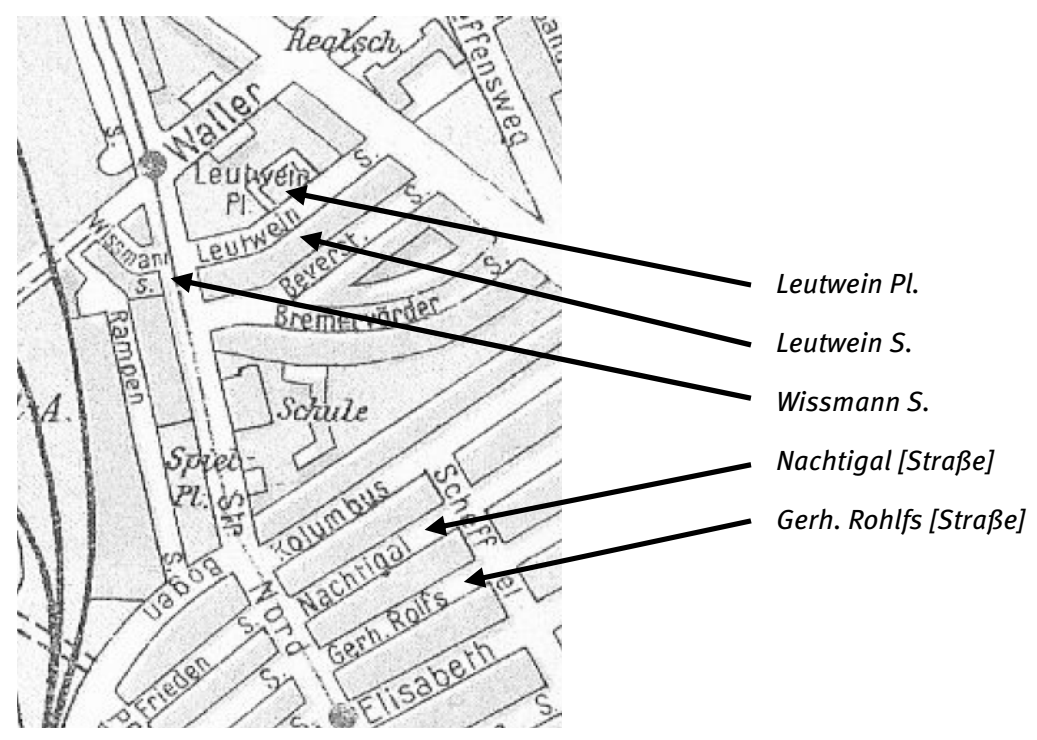

Abb. 4: Stadtplan Bremen (1927), Ausschnitt Waller Vorstadt.

Zeitgenössische Stadtpläne sind, wenngleich in einer geringeren Zahl als Adressbücher, in digitalisierter Form online verfügbar: Neben Digitalisierungsprojekten lokaler Einrichtungen ${ }^{80}$ ist insbesondere das vom privaten Landkartensammler M. Ritz erstellte Landkartenarchiv mit mehr als 13.000 online zur Verfügung stehenden Karten hervorzuheben. ${ }^{81}$ Für den Untersuchungszeitraum der vorliegenden Studie weist das Landkartenarchiv bspw. nahezu 400 historische Stadtpläne für diverse Städte des Deutschen Reichs bis 1945 auf. Zusätzlich wurde die umfangreiche Stadtplansammlung der Staatsbibliothek zu Berlin Preußischer Kulturbesitz (SBBPK) genutzt, die in der dafür zuständigen Kartenabteilung für einen Großteil des Ortskorpus zeitgenössische Pläne in zeitlichen Staffelungen von 1884 bis 1945 aufweist. ${ }^{82}$

80 Man vgl. dazu bspw. das Historische Portal Essen (https://historischesportal.essen.de/ startseite_7/historisches_portal_startseite.de.jsp, Abruf am 28/06/2019).

81 http://www.landkartenarchiv.de/, Abruf am 28/06/2019.

82 https://staatsbibliothek-berlin.de/die-staatsbibliothek/abteilungen/karten/, Abruf am 28/06/2019. 


\subsection{Identifizierungs- und Inventarisierungsaufgabe anhand ausgewählter Städte des Ortskorpus}

Der Arbeitsschritt erforderte die systematische Überprüfung aller Groß- und Mittelstädte des hierfür angelegten Ortskorpus auf etwaige kolonial motivierte Straßennamenverfügungen zwischen 1884 und 1945, das nur durch Hinzuziehung der zuvor beschriebenen unterschiedlichen Quellengattungen erfolgreich bewältigt werden kann. Jene Identifizierungs- und Inventarisierungsarbeiten sollen für die zwei im erstellten Ortskorpus vertretenen Großstädte Dresden und Breslau [Wrocław] exemplarisch aufgezeigt werden.

\subsubsection{Kolonial motivierte Straßenbenennungen in Dresden}

Die Vergabepraxis von Lüderitzstraße und Wissmannstraße für zwei zur Kolonialzeit neu erschlossene Straßenzüge in Dresden wurden erfreut von der DKG kommentiert (vgl. DKZ 22.3.1913: 201, Kap. 1). Beide SN-Token beziehen sich auf Kolonialakteure des Deutschen Kaiserreichs. Ein Straßennamenlexikon liegt für Dresden nicht vor. Die Sichtung eines Dresdner Einwohnerbuchs aus nationalsozialistischer Zeit (vgl. Adressbuch Dresden 1940 V) ergibt, dass neben den beiden Benennungen fünf weitere Straßennamen bis 1945 verfügt wurden, deren Erstglieder auf Akteure der deutschen Kolonialepoche (Karl-Peters Straße, Leutweinstraße, Rohlfsstraße) und auf errichtete Verwaltungszentren in den kolonisierten Räumen (Swakopmunder Straße, Windhuker Straße) referieren. Alle Personen- und Ortsnamen sind in den Koloniallexika (vgl. Kausch 1903, Schnee 1920) mit eigenen Einträgen aufgelistet. Aus dem Straßenverzeichnis geht hervor, dass sieben SN-Token im Stadtteil Räcknitz-Zschertnitz zu verorten sind. Anhand eines am Ende der 1930er Jahre erstellten Stadtplans ergibt sich die zeitgenössische Verfügung der Namen in den öffentlichen Raum als Kolonialcluster (siehe Abb. 5).

Es handelt sich um eine thematisch kohärente Benennungspraxis von neu erschlossenen Straßen, die parallel zueinander angelegt wurden oder sich unmittelbar kreuzen. Die vergleichende Sichtung älterer Stadtpläne ergibt, dass es sich bei den kolonial motivierten Namen nicht um einen einmaligen Benennungsakt handelt, sondern dass das Kolonialcluster hinsichtlich der jeweiligen Namenverfügungen einer zeitlichen Schichtung unterliegt; im Stadtplan von 1923 sind nur die unmittelbar südlich an den Park angrenzenden Benennungen aufgeführt (siehe Abb. 6). 


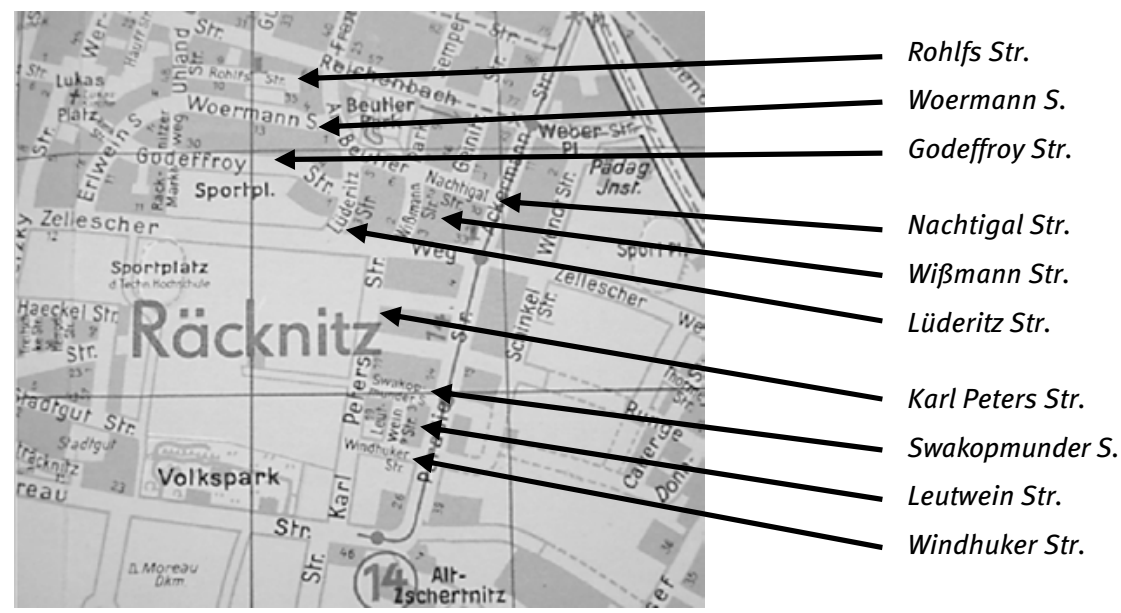

Abb. 5: Stadtplan Dresden (1939), Ausschnitt Räcknitz/Zschertnitz.

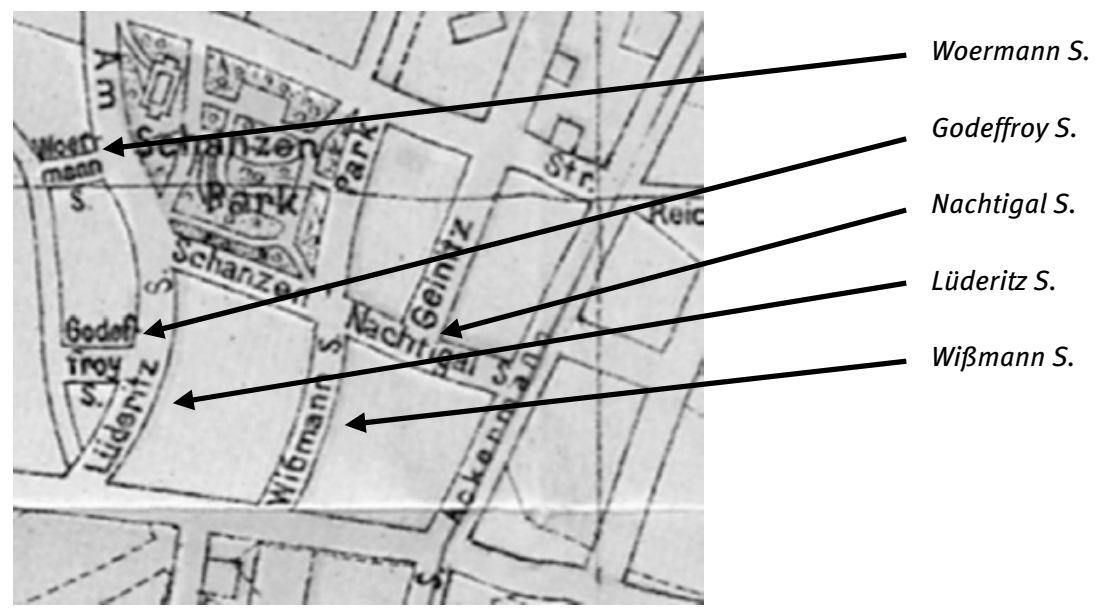

Abb. 6: Stadtplan Dresden (1923), Ausschnitt Räcknitz/Zschertnitz.

Neben den bereits 1913 erfolgten Benennungen Lüderitzstraße und Wissmannstraße sind drei weitere SN-Token verzeichnet, die ebenfalls auf Akteure der deutschen Kolonialepoche referieren (Woermannstraße, Godeffroystraße und Nachtigalstraße). Sie wurden gegen Ende der faktischen Kolonialzeit oder kurz danach 
administrativ verfügt. ${ }^{83}$ Für den Untersuchungszeitraum liegen Ortsgesetzblätter, also rechtsgültige Satzungen und ordnungsbehördliche Verordnungen ${ }^{84}$ vor, in denen unter anderem Bekanntmachungen der königlichen Polizeidirektion desselbigen Jahres versammelt sind. Aus dem Dresdner Ortsgesetzblatt von 1913 geht hervor, dass neben den SN-Token Lüderitzstraße und Wißmannstraße auch die Benennungen Woermannstraße, Godeffroystraße und Nachtigalstraße für unmittelbar angrenzende, neu angelegte Straßenzüge erfolgten.

70. Bekanntmachung über Straßenbenennungen. [Fettdruck im Original] [...] Mit Allerhöchster Genehmigung haben wir die Benennung weiterer Straßen [...] beschlossen.

I. die Straße 1 zwischen Uhlandstraße und Schanzenpark Woermannstraße, zum Andenken an den Hamburger Großkaufmann und Reeder Adolf Woermann, Besitzer von Faktoreien und Plantagen in Kamerun, Mitbegründer der Woermann-Dampferlinie nach Westafrika;

II. die Straße 2 zwischen dem Lukasplatz und der Lüderitzstraße Godeffroystraße, zum Andenken an den Hamburger Großkaufmann Jh. Cesar Godeffroy, Begründer von Handelsstationen und Plantagen in der Südsee;

III. die Straße 3 zwischen der Wißmann- und Ackermannstraße Nachtigalstraße, zum Andenken an den Afrikaforscher Gustav Nachtigal, der als Generalkonsul Togo, Kamerun und Lüderitzland unter deutschen Schutz stellte. (Dresdner Ortsgesetzblatt 1913: 50-51)

Bis 1913 ist die Verfügung eines Kolonialclusters festzustellen, mit dem das „unvergessene[s] Heldentum“ (Deutscher Kolonialkrieger-Bund 1924) im Raum der deutschen Metropole geehrt und/oder gewürdigt werden sollte. Alle fünf Benennungen erfolgten bereits in der de facto-Kolonialzeit (vgl. Parzellierungsplan vom Gelände der Terraingesellschaft Dresden-Süd: ca. 1914). Die Straßenzüge der in jüngerer Zeit erfolgten kolonialzeitbezogenen Benennungen südlich des Zelleschen Weges sind auf dem Bebauungsplan noch nicht angelegt: Bei Karl-Peters Straße, Leutweinstraße, Rohlfsstraße, Swakopmunder Straße und Windhuker Straße handelt es sich um thematisch kohärente Benennungen, die in die Zeit des Nationalsozialismus fallen. ${ }^{85}$ Die administrativen Verfügungen des Dresdner Rats sind datiert auf das Jahr 1934 (vgl. Dresdner Ortsgesetzblatt 1934: 14), die Rohlfsstraße wird vier Jahre später durch den Oberbürgermeister verfügt (vgl. Dresdner Ortsgesetzblatt 1938: 7). Das bis 1945 verfügte Kolonialcluster unterliegt also hinsichtlich seiner Benennungen einer zeitlichen Schichtung: Benennungen in der Kolonial-

83 Die entsprechenden kolonialen SN-Token sind bis 1911 weder in Straßennameverzeichnissen der Adressbücher (Adressbuch Dresden 1910 III) noch in Stadtplänen (Dresdener Stadtplan 1911) verzeichnet.

84 Man vgl. dazu https://www.guetersloh.de/de/rathaus/veroeffentlichungen/gesammeltesortsrecht.php\#anchor_7eb0f698_Accordion-Bauwesen, Abruf am 28/06/2019.

85 Die entsprechenden SN-Token sind noch nicht im Straßenverzeichnis des Dresdner Adressbuchs von 1932 (III) aufgeführt. 
zeit wurden durch die jüngere Vergabe einer ganzen Reihe weiterer Namen im Zuge der fortschreitenden infrastrukturellen Erschließung themenkohärent fortgeführt. Bis 1945 ist ein Großcluster mit zehn nachweislich in kolonialer Motivik verfügten Straßennamen festzustellen, die auf Kolonialakteure des Kaiserreichs und Orte in den kolonisierten Räumen referieren.

\subsubsection{Koloniale motivierte Straßenbenennungen in Breslau [Wroctaw]}

Auch für die Hauptstadt der Provinz Schlesien können bis 1945 kolonial motivierte Straßennamenverfügungen erhoben werden. Im Straßenverzeichnis des zu Beginn der 1940er Jahre herausgegebenen Einwohnerbuchs sind drei SNToken für den Stadtteil Mochbern aufgelistet, die in einem unmittelbaren Bezug zur deutschen Kolonialgeschichte stehen:

Heinrich-Schnee-Straße (Mochbern): [...] Heinrich Schnee, ehem. Gouverneur von DeutschOst-Afrika. [...] Die hier liegenden Grundstücke gehören zur Apiastr., Dualastr., Samoastr. und Tangastr. [...]. (Adressbuch Breslau 1943 II: 136)

Lettow-Vorbeck-Straße (Mochbern) [...] Paul v. Lettow-Vorbeck. Kommandeur der Schutztruppe in Deutsch-Ostafrika 1914-1918. (Adressbuch Breslau 1943 II: 207)

Wissmannstraße (Mochbern) [...] Herm. v. Wißmann, Afrikareisender, erwarb Deutsch-Ostafrika für Deutschland. (Adressbuch Breslau 1943 II: 397)

Der Eintrag für das SN-Token Heinrich-Schnee-Straße informiert über die Benennungen der unmittelbar angrenzenden Straßenzüge (Apia-, Duala-, Samoaund Tangastraße), die sich auf Orte in den Kolonialgebieten beziehen. Für die jeweiligen SN-Token sind im Straßenverzeichnis entsprechende Einträge für Mochbern verzeichnet, allerdings ohne Angabe von Benennungsmotiviken. Zudem können für diesen Stadtteil weitere Namenartikel identifiziert werden, deren Erstglieder sich in kommemorativer Intention auf Personen (Karl-PetersStraße, Lüderitzstraße) und Orte (Togostraße, Windhukstraße) der deutschen Kolonialepoche beziehen. Für alle Erstglieder der betreffenden Konstruktionen sind Einträge in den Koloniallexika (vgl. Kausch 1903, Schnee 1920) vorhanden. Die Annahme eines in den öffentlichen städtischen Raum verfügten Kolonialclusters mit elf kolonial motivierten SN-Token wird durch die Konsultation zeitgenössischer Stadtpläne eindeutig bestätigt (Abb. 7).

Bei den kolonialen Straßennamen handelt es sich um administrative Verfügungen der 1930er Jahre. Sie tauchen in älteren Stadtplänen (vgl. Stadtplan 1906, 1911, ca. 1930) noch nicht auf. Das in Breslau [Wrocław] verfügte Kolonialcluster unterliegt also hinsichtlich der Verfügungszeiträume der jeweiligen SN- 
Token - im Gegensatz zu dem Dresdener Kolonialviertel - keiner zeitlichen Schichtung. Alle Namen wurden über ein Jahrzehnt nach der de facto-Kolonialzeit des Kaiserreichs verfügt.

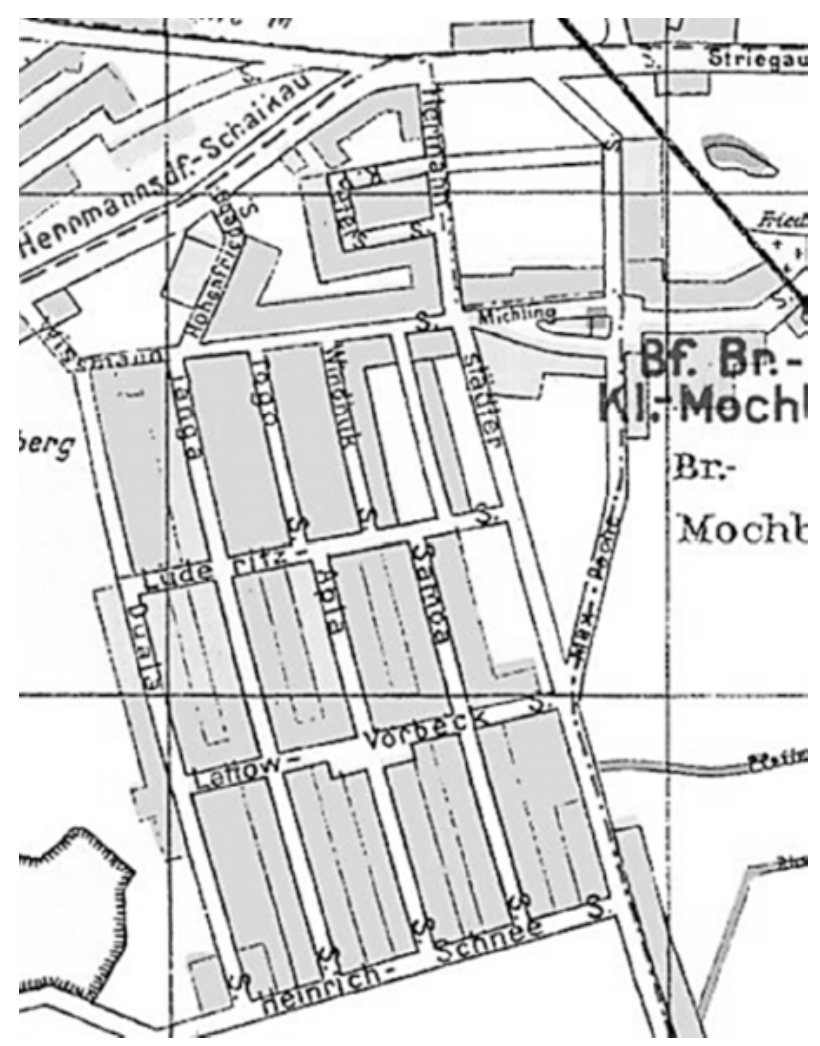

Abb. 7: Stadtplan Breslau (etwa 1941), Ausschnitt Mochbern.

\subsection{Ergebnisse}

Die Zusammenstellung kolonial motivierter Straßennamen für zwei Großstädte des Ortskorpus ergibt 21 SN-Token, die anhand der Sichtung von administrativen Akten, Adressbüchern und Stadtplänen in historischen Zeitschnitten für den Untersuchungszeitraum erhoben werden konnten. Die Inventarisierungsaufgabe zeigt bereits anhand der zwei Einzelorte, dass die methodische Vorgehensweise der additiven Informationsgewinnung aus den jeweils verfügbaren 
historischen Quellen und Quellengattungen ein wesentliches und zugleich erfolgreiches Verfahren für die Identifizierung kolonialer Namen darstellt. Gleichzeitig wird deutlich, dass die Aufarbeitung kolonialer Benennungspraktiken zur Untersuchung der damit versprachlichten kolonisatorischen Selbstzuschreibungskonzepte im öffentlichen Raum der deutschen Metropole durch die Perspektive ex ante aufgearbeitet werden muss: Das aktuelle Dresdener Straßenverzeichnis gibt keinerlei Hinweise auf die Verfügung eines Kolonialclusters in der ersten Hälfte des 20. Jahrhunderts; von der historischen Clusterbenennung mit über zehn Kolonialismen für Straßenzüge in nächster Nähe ist im rezenten Namenbestand der Stadt nur noch das SN-Token Rohlfsstraße als gegenwärtiges Beibehaltungsprodukt verzeichnet. Im aktuellen Straßenverzeichnis der Großstadt Wrocław ${ }^{86}$ sind keine etwaigen polnischen Übersetzungsprodukte der historischen Kolonialismen aus Zeiten der Zugehörigkeit zum Deutschen Reich festzustellen. Die Aufarbeitung von Benennungspraktiken im Kontext kolonialer Raumaneignung bzw. -besetzung in der deutschen Metropole selbst kann durch Inventarisierungsarbeiten rezenter Straßennamenbestände nicht geleistet werden. Sie erfordert die systematische Zusammenstellung kolonialer Straßennamen auf Basis ihrer historischen Benennung, die sodann nach Strukturmustern und Diskursfunktionen zu analysieren sind. Kontrastive Studien, die sodann Umbenennungspraktiken von kolonialen Mikrotoponymen in der zweiten Hälfte des 20. Jahrhunderts bis heute systematisch aufarbeiten, können erst in einem zweiten Schritt erfolgen: Sie sind dann realisierbar und aussagekräftig, wenn die historischen Benennungsprozesse bis 1945 ortsübergreifend erhoben und nach toponomastischen und koloniallinguistischen Fragestellungen ausgewertet wurden, „denn nur auf der Grundlage eines vergleichbar ermittelten Datensets lassen sich unseres Erachtens ja überhaupt erst Weiterungen der Namengeschichte angemessen verfolgen und einordnen“ (Stolz und Warnke 2017: 211).

Auf eine etwaige gegenwartssprachliche Perspektive, wie sie derzeit zu Umbenennungsforderungen und -aktivitäten in einzelnen deutschen Städten geführt werden, wird in dieser Studie bewusst verzichtet. Dass sich das in der vorliegenden Arbeit zusammengestellte Inventar aufgrund der unterschiedlichen Fragestellungen und Vorgehensweisen deutlich von den appellativ angestrebten Ansätzen gesellschaftspolitischer Akteursgruppen unterscheidet, die sich vor dem Hintergrund ihres Interesses an postkolonialer Erinnerungskultur mit rezenten Straßennamen beschäftigen, soll an einem Beispiel gezeigt werden: Der Verein Berlin Postkolonial e. V. stellt eine „Übersicht der bundesdeutschen Städte und Ort-

86 http://geoportal.wroclaw.pl/www/en/index.shtml, Abruf am 28/06/2019. 
schaften, in denen koloniale Straßennamen zu finden sind“, zur Verfügung. Die Behandlung aktueller Straßennamen erfolgt mit aufklärerischem und positionsbeziehendem Charakter. ${ }^{87}$ Postkolonial.de-Gruppierungen stellen in der Regel semantische gegenwartssprachliche Bezüge zu rezenten Straßennamen her; eine strenge Definition, welche Kriterien für die Zuschreibung eines Straßennamens zum ,kolonialen` oder ,postkolonialen` Namen erfüllt sein müssen, hängt dabei nicht primär von der Motivik der historischen Namenverfügung seitens der zeitgenössischen Administration ab. Schulz und Ebert (2017: 168-169) zeigen am Beispiel der Benennung Konrad-Adenauer-Platz in Freiburg i. B. auf, dass der rezente Straßenname innerhalb des postkolonialen Aktivismus als „koloniale[r] Straßenname[n] in und um Freiburg“ ${ }^{* 88}$ aufgeführt wird. Für das zu erstellende sprachhistorische Inventar der vorliegenden Studie spielt das SN-Token keine Rolle: Adenauer war gegen Ende der Weimarer Republik stellvertretender Präsident der DKG. Die administrative Benennung erfolgte posthum gegen Ende der 1960er Jahre, und zwar zu Ehren seines Bundeskanzleramtes in der BRD. Neben der Tatsache, dass die Namenverfügung nicht in den Untersuchungszeitraum dieser Arbeit fällt, ist darüber hinaus auch keine etwaige koloniale Benennungsmotivik gegeben, die sich auf sein kolonialrevisionistisches Bestreben zu Beginn der 1930er Jahre richtet.

Ein weiteres Beispiel soll diese abgrenzende Kategorisierung vertiefen: Historische Straßennamenverfügungen, die Joachim Nettelbeck ehren und/oder würdigen sollten, sind noch im rezenten Bestand deutscher Städte vorzufinden; postkoloniale Gruppierungen listen für 21 bundesdeutsche Städte Konstruktionen mit dem anthroponymischen Erstglied Nettelbeck auf, die damit noch heute den „Kolonialverbrecher würdigen“. ${ }^{89}$ Der pommersche Seemann Joachim Nettelbeck verstarb bereits im Jahr 1824 (vgl. Vogt 1999: 83-84). Nichtsdestotrotz wird er in einem Artikel der DKG über sechzig Jahre nach seinem Tod als Vorkämpfer für eine Deutsche Kolonialpolitik inszeniert (vgl. Katterfeld 1886: 170-174). Zur Person ist aber kein Eintrag in den einschlägigen Koloniallexika (vgl. Kausch 1903, Schnee 1920) verzeichnet. Die Überprüfung der 21 Token hinsichtlich etwaiger kolonialer Benennungsmotiviken zum historischen Verfügungszeitpunkt ergibt, dass nur ein singuläres SN-Token zum Inventar der vorliegenden Arbeit aufzunehmen ist; nur die in München verfügte Benennung sollte unmittelbar auf sein zeitlebens koloniales Engagement (fett markiert) referieren:

87 „Zu einem grundlegenden Wandel im Umgang mit Deutschlands kolonialem Erbe, zur Umbenennung von Straßen, die koloniale Akteure ehren sowie zur Förderung postkolonialer Erinnerungskulturen“ (http://freedom-roads.de/frrd/willkom.htm, Abruf am 28/06/2019).

88 http://www.freiburg-postkolonial.de/Seiten/strassen.htm, Abruf am 28/06/2019.

89 http://www.freedom-roads.de/frrd/staedte.htm, Abruf am 28/06/2019. 
Nettelbeckstraße: Joachim Nettelbeck, Seefahrer, organisierte mit Schill und Gneisenau erfolgreich die Verteidigung der von den Franzosen 1806 belagerten Festung Kolberg; er weist als einer der ersten auf die Notwendigkeit hin, Kolonien zur Stützung der Landmacht zu erwerben. (Adressbuch München 1940 IV: 454)

Die Namenvergabe erfolgte in nationalsozialistischer Zeit in MünchenBogenhausen, und zwar zeitgleich mit weiteren SN-Token, die in kommemorativer Intention auf Kolonialakteure aus der Zeit des Kaiserreichs referieren sollten. Auch das SN-Token Nettelbeckstraße ist aufgrund seines Verfügungszeitpunktes, der Verortung des Straßenzugs innerhalb des Kolonialclusters und der im Einwohnerbuch angegebenen Benennungsmotivik als zeitgenössisch kolonialer Straßenname zu bewerten. In allen anderen 20 Fällen sind keine Anhaltspunkte für eine etwaige koloniale Motivik im Zuge der Verfügung ermittelbar: Sowohl die historische Benennungsakten als auch die Einwohnerbücher legen ausschließlich Motiviken dar, die sich auf Nettelbecks entscheidenden Anteil an der Verteidigung seiner preußischen Heimatstadt Kolberg gegen französische Truppen beziehen. ${ }^{90}$ Und auch keines der $20 \mathrm{SN}$-Token ist innerhalb klein oder groß angelegter Kolonialcluster ermittelbar. Nur das SN-Token Nettelbeckstraße in MünchenBogenhausen ist ins Namenkorpus der vorliegenden Arbeit aufzunehmen: Für diesen einzelortsbezogenen Fall liegt eine unübliche Instrumentalisierung der Person vor, was durch die historische Benennungsmotivik des historischen Adressbucheintrags und der Analyse raumsemantischer Muster, die durch das Cluster entstehen, nachgewiesen werden konnte.

Es lassen sich weitere Beispiele anfügen, die den unterschiedlichen sprachhistorischen Inventarbestand der vorliegenden Untersuchung im Vergleich zu den von gesellschaftspolitischen Gruppierungen fokussierten Straßennamen untermauern. So wurden Straßenbenennungen, die in kommemorativer Intention auf den Grafen von Waldersee referieren sollten und noch im rezenten Bestand deutscher Städte vorzufinden sind, nicht ins kolonial motivierte Nameninventar der vorliegenden Arbeit aufgenommen..$^{91}$ Zeitgenössische Benennungsmotiviken solcher posthum erfolgten Namenverfügungen nehmen folgende Zuschreibungen vor:

90 Bspw. Dortmund: „Nettelbeckstraße [...] bekannt durch seine heldenmütige Verteidigung Kolbergs gegen die Franzosen im Jahre 1807.“ (Adressbuch Dortmund 1938 III: 204). Erfurt: „Nettelbeckufer [...] Verteidiger von Kolberg 1806/1807“ (Adressbuch Erfurt 1924 IV: 135). Wuppertal: „Nettelbeckweg [...] nach dem Verteidiger der Feste Kolberg benannt“ (Adressbuch Wuppertal 1938 III: 289).

91 Der Verein Berlin Postkolonial e. V. listet dagegen sechs Straßennamen mit dem Erstglied Waldersee auf (http://www.freedom-roads.de/frrd/staedte.htm, Abruf am 28/06/2019). 
Walderseestraße: Preußischer General. (Adressbuch Bremen 1919 III: 746)

Walderseestraße: Alfred von Waldersee, preußischer Generalfeldmarschall, geb. 8.4.1832, gest. 5.3.1904; Oberbefehlshaber in China während der chinesischen Wirren. (Adressbuch Dortmund 1938 III: 294)

Walderseestraße: Benannt zu Ehren des Grafen von Waldersee, dessen Korps-Kommando die Lübecker Garnison 1891 bis 1898 unterstandt [sic!] - Moltkeplatz. (Adressbuch Lübeck 1939 III: 639)

Wenngleich der preußische General bei der Niederschlagung der in zeitgenössischen Quellen als „Boxeraufstand“ (Schnee 1920 I: 236) bezeichneten Strafexpeditionen beteiligt war, ist keine spezifisch koloniale Benennungsmotivik für die ortsübergreifenden Verfügungen derartiger SN-Token zu konstatieren. Für den Graf von Waldersee sind in beiden Koloniallexika (vgl. Kausch 1903, Schnee 1920) keine separaten Einträge vorhanden. Aus der Überprüfung historischer Stadtpläne kann darüber hinaus festgestellt werden, dass die relevanten Konstruktionen weder innerhalb noch in nächster Nähe von Kolonialclustern zu verorten sind. Stattdessen wurden derartige Namenmuster nicht selten zusammen mit SN-Token verfügt, die sich auf weitere preußische Generäle bezogen.

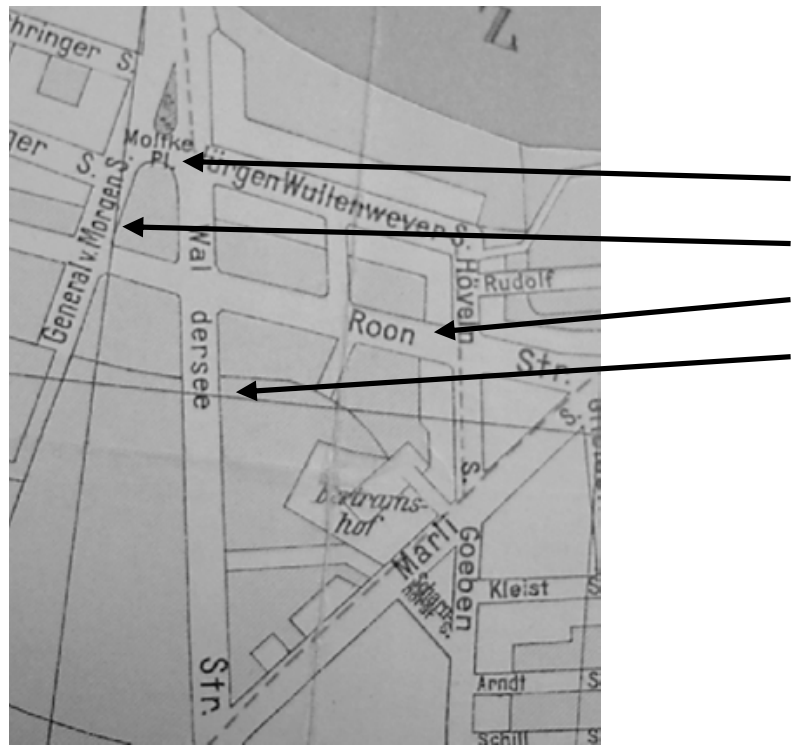

Moltke Pl.

General v. Morgen S.

Roon Str.

Waldersee Str.

Abb. 8: Stadtplan Lübeck (1938). 
Bei dem noch im rezenten Bestand vorliegenden SN-Token Karolinenstraße in Neustadt an der Haardt bzw. Weinstraße, das der Verein Berlin Postkolonial e. V. zu „Straßennamen nach kolonialen Erinnerungsorten“92 aufgrund des toponymischen Kerns Karolinen mit Bezug auf das Inselgebiet Deutsch-Neuguineas zählt, handelt es sich allerdings um ein Missverständnis, das durch quellengestützte philologische Analyse leicht aufzuklären ist: Die Mittelstadt gehört zum erstellten Ortskorpus und wurde daher auf etwaige kolonial motivierte Benennungen untersucht. Im rezenten Namenbestand sind die Straßennamen GustavNachtigal-Straße, Karl-Peters-Straße, Lüderitzstraße und Von-Wissmann-Straße für unmittelbar angrenzende Straßenzüge ermittelbar, deren Erstglieder sich allesamt auf Kolonialakteure in der Zeit des Kaiserreichs beziehen. Die Konstruktionen wurden 1938 als Kolonialcluster verfügt; sie sind ebenfalls bei Berlin Postkolonial e. V. aufgelistet. Da das verfügbare Straßenverzeichnis von 1938 alle Benennungen des vergangenen Jahres auflistet, sind die vier Kolonialismen noch nicht vorzufinden. Die Karolinenstraße dagegen schon (vgl. Adressbuch Neustadt an der Weinstraße 1938 III: 61). Eine Konsultation älterer Stadtpläne zeigt, dass die Benennung Karolinenstraße sogar Jahre zuvor administrativ verfügt wurde: Im Plan von 1912 ist das Gebiet des heutigen Kolonialviertels noch unbebaut, während die SN-Token Karolinenstraße und Amalienstraße für den östlich dazu angrenzenden Straßenzug bereits verzeichnet sind. Bis zu Beginn der 1930er Jahre bleibt diese Raumsituation unverändert (vgl. Stadtplan Neustadt an der Haardt 1931/32). Der Verdacht, dass die an das spätere Kolonialcluster anschließende Karolinenstraße in keinem kolonialen Zusammenhang verfügt wurde, hat sich bestätigt: Eine Recherche im Kontakt mit dem Stadtarchiv ergab, dass die zeitgleichen Benennungen Karolinenstraße und Amalienstraße nach zwei weiblichen Familienangehörigen berühmter Neustadter Persönlichkeiten aus dem 19. Jahrhundert erfolgte:

Ehrenbürger Hauber legte in den 1880er Jahren den Karolinenhain an [...]. Auf einem [...] unbehauenen Sandstein steht „Karolinenruhe“ in Erinnerung an seine Frau Karoline Hauber. Nach ihr heißt auch die Karolinenstraße, die 1884 gebaut wurde. (Die Rheinzeitung 2001 Nr. 63, 14.03.2001)

Der Benennung Karolinenstraße kann (genauso wie der der Amalienstraße) eine eindeutige lokalhistorische Motivik zugeschrieben werden. Sie ist für das in dieser Arbeit zusammengestellte Nameninventar eindeutig auszuschließen. Das Beispiel zeigt, dass Berlin Postkolonial e. V. nicht nur eine vorrangig gegenwartssprachliche Bewertung des rezenten Namenbestandes vornimmt und zu

92 http://www.freedom-roads.de/frrd/staedte.htm, Abruf am 28/06/2019. 
einer unterschiedlichen Zuordnung und Bewertung von sekundären Namen kommt, sondern dass dabei auch Fehlschlüsse konstatiert werden können. Im Hinblick auf Namenmustern bei entsprechend gleichen ausdrucksseitigen Erstgliedern vergegenwärtigt es auch, wie behutsam bei der sprachhistorischen Identifizierung kolonial motivierter Straßennamen innerhalb themenkohärenter Cluster als Endprodukte einer prozessualen, sich im Laufe von Jahren oder sogar Jahrzehnten weiterentwickelnden Linguistic Landscape vorgegangen werden muss: Für das erstellte Ortskorpus konnte die Konstruktion mit dem Erstglied Karolinen, die sich zum historischen Benennungszeitpunkt nachweislich in kolonialer Intention auf das kolonisierte Inselgebiet bezieht, nur ein einziges Mal für die Stadt Heilbronn (Karolinenweg) erhoben werden (Kap. 3.3.4, Abb. 27). Es handelt sich um ein singuläres Phänomen und keinesfalls um eine prototypische Konstruktion kolonial motivierter Namenvergabeprozesse bis 1945. Hier zeigt sich aber zugleich deutlich, dass die intensive sprachhistorische Analyse der Benennungen der Forderung nach Umbenennungen unbedingt vorausgehen muss, will man Unsicherheiten wie diese vermeiden.

Die vorigen Beispiele demonstrieren für die Erstellung des sprachhistorischen Inventars kolonialer Mikrotoponyme folgende methodische Anforderungen: Die Frage, ob ein zwischen 1884-1945 verfügter kommemorativer Straßenname ,kolonial' ist, kann nur mithilfe der Aufdeckung inhaltsseitiger Kriterien beantwortet werden und erfordert die durch empirisch-philologisch abgesicherte Analysen herauszufindende Motivik der Namen zum Verfügungszeitpunkt. Hierfür müssen verschiedene historische Quellen und Quellengattungen (Kap. 3.3) zur additiven Informationsgewinnung genutzt werden. Die Aufdeckung raumsemantischer Muster (Kolonialcluster) durch die Hinzuziehung von Stadtplänen in diachronen Staffelungen stellt ein Hilfsmittel für die Inventarisierungs- aber auch die nötigen Abgrenzungsarbeiten für das zu erstellende koloniale Nameninventar dar. Die fundierte koloniale Zuschreibung muss für jedes einzelne SN-Token geprüft werden: Konstruktionen mit ausdrucksgleichen Erstgliedern müssen, selbst wenn letztere in den zeitgenössischen Koloniallexika aufgeführt sind, immer tokenbezogen hinsichtlich ihrer zeitgenössischen Benennungsmotivik nachvollzogen werden (vgl. Petersstraße in Leipzig, Karolinenstraße in Neustadt a. d. Haardt bzw. Weinstraße). Die vorliegende Arbeit begibt sich - im Gegensatz zu den gesellschaftspolitisch forcierten postkolonial.de-Gruppen - auf keine aus gegenwartssprachlicher Perspektive angelegte Spurensuche des deutschen Kolonialismus (vgl. Schulz 2019). Derartige Gruppierungen sprechen dem rezent vorliegenden Straßennamenbestand mit einem appellativ intendierten Ansatz koloniale Semantiken auf der Inhaltsseite zu, um zu Umbenennung und Diskussion diverser Namen aufzurufen. Aus der Perspektive der postkolonial.de-Gruppierungen und 
den damit verbundenen gesellschaftspolitischen Intentionen mag der präskriptive, gegenwartssprachliche Ansatz angebracht sein. Für die vorliegende sprachwissenschaftliche Untersuchung, die Kolonialismen für einen historischen Beobachtungszeitraum erhebt und in Bezug auf Namenstrukturen sowie diskursive Funktionen analysiert, ist ein solcher präskriptiv vorgehender Ansatz nicht zulässig: „Es ist [...] der Effekt einer dominanten Spielart der Linguistik, die sich durchaus wohl damit fühlt, nicht in interventionalistische, politische Diskurse eingebunden zu sein“ (Warnke et al. 2016: 13). 